\title{
Asynchronous inputs alter excitability, spike timing, and topography in primary auditory cortex
}

\author{
Pritesh K. Pandya *, Raluca Moucha, Navzer D. Engineer, Daniel L. Rathbun, \\ Jessica Vazquez, Michael P. Kilgard \\ Neuroscience Program, School of Behavioral and Brain Sciences, PO Box 830688, GR 41, University of Texas at Dallas, \\ Richardson, TX 75083-0688, USA
}

Received 6 February 2004; accepted 30 November 2004

Available online 28 December 2004

\begin{abstract}
Correlation-based synaptic plasticity provides a potential cellular mechanism for learning and memory. Studies in the visual and somatosensory systems have shown that behavioral and surgical manipulation of sensory inputs leads to changes in cortical organization that are consistent with the operation of these learning rules. In this study, we examine how the organization of primary auditory cortex (A1) is altered by tones designed to decrease the average input correlation across the frequency map. After one month of separately pairing nucleus basalis stimulation with 2 and $14 \mathrm{kHz}$ tones, a greater proportion of A1 neurons responded to frequencies below $2 \mathrm{kHz}$ and above $14 \mathrm{kHz}$. Despite the expanded representation of these tones, cortical excitability was specifically reduced in the high and low frequency regions of $\mathrm{A} 1$, as evidenced by increased neural thresholds and decreased response strength. In contrast, in the frequency region between the two paired tones, driven rates were unaffected and spontaneous firing rate was increased. Neural response latencies were increased across the frequency map when nucleus basalis stimulation was associated with asynchronous activation of the high and low frequency regions of A1. This set of changes did not occur when pulsed noise bursts were paired with nucleus basalis stimulation. These results are consistent with earlier observations that sensory input statistics can shape cortical map organization and spike timing.
\end{abstract}

(C) 2004 Elsevier B.V. All rights reserved.

Keywords: Cortical plasticity; Map reorganization; Activity-dependent Hebbian plasticity; Sensory input; Rat; Auditory cortex; Nucleus basalis

\section{Introduction}

Under appropriate conditions, adult cortical sensory representations can be remodeled through learning (Jenkins et al., 1990; Recanzone et al., 1992a, 1993). Experiments conducted in the visual, somatosensory, and auditory systems have provided compelling evidence that different sensory input patterns can lead to distinctly different forms of cortical reorganization (Allard et al., 1991; Kilgard et al., 2002; Stryker and Strickland, \footnotetext{
2491.

Corresponding author. Tel.: +1 972883 2345/76; fax: +1 972883

E-mail address: pritesh@utdallas.edu (P.K. Pandya).
}

1984; Wang et al., 1995). Behaviorally relevant sensory and motor events are marked by increased activity in nucleus basalis (NB), which projects to the entire cortex and powerfully modulates plasticity (Conner et al., 2003; Kilgard and Merzenich, 1998b; Kilgard et al., 2001a; Weinberger, 1998).

Changes in neural connectivity and dynamics likely provide the biological basis for learning and memory (Kandel, 2001; Katz and Shatz, 1996). Synaptic connections typically follow correlation-based learning rules such that connections between neurons with correlated activity are strengthened and connections between uncorrelated neurons are weakened (Bi and Poo, 2001; Hebb, 1949; Stent, 1973). These changes in synaptic 
strength are often accompanied by changes in neural sensitivity and latency.

Observations from several cortical plasticity studies are consistent with the operation of correlation-based learning rules in vivo. Increasing input correlation via strobe rearing, whisker pairing protocols, or tactile discrimination training results in shorter latencies in visual and somatosensory cortices (Armstrong-James et al., 1994, 1998; Recanzone et al., 1992b). In contrast, decreasing input correlation via strabismus, monocular deprivation, or frequency discrimination training results in prolonged latencies in the visual and auditory cortices (Chino et al., 1988; Chino et al., 1983; Eschweiler and Rauschecker, 1993; Recanzone et al., 1993). Decreased response latencies were observed after a single tone was paired with nucleus basalis stimulation, while latencies were increased when seven different tones were independently paired with nucleus basalis stimulation (Kilgard et al., 2001a). In that study, we speculated that latency was decreased when map expansion drove a net increase in the correlated activity across A1 and latency was increased when tonal inputs were distributed across A1 in the absence of map plasticity. Collectively, these observations are compatible with the hypothesis that synaptic correlation-based learning rules have a significant impact on the expression of network level plasticity.

To confirm that a net decrease in input correlation induces a weakening of auditory cortical responses that is reflected by longer response latencies and reduced response strength, we exposed animals to tonal inputs designed to activate two non-overlapping neural populations at different times. These tones were paired with electrical activation of the nucleus basalis as in our earlier studies. Nucleus basalis stimulation paired with sensory input has been used extensively by several research groups to promote input guided cortical plasticity in the absence of behavior (Bakin and Weinberger, 1996; Edeline et al., 1994; Kilgard et al., 2001a; Metherate and Ashe, 1993; Rasmusson and Dykes, 1988). While nucleus basalis stimulation is an unnatural method for gating representational plasticity, this paradigm makes it possible to manipulate sensory statistics by delivering arbitrary input patterns without the need for time-consuming behavioral training or surgical procedures.

In the present study, we use this well-established technique to further explore how asynchronous activation directs plasticity in primary auditory cortex. We report that pairing randomly interleaved high and low frequency tones, which drove a net decrease in the correlation across the frequency map, with activation of neuromodulatory inputs led to map plasticity, decreased cortical excitability, increased spontaneous activity, and lengthened response latencies compared to naive controls. This set of changes did not occur when pulsed noise bursts were paired with nucleus basalis stimulation. Our results extend earlier observations that spatial and temporal cortical network organization can be shaped by distributed sensory inputs.

\section{Methods}

The neural responses presented in this report were obtained from nine NB-stimulated rats and 15 naive controls. Detailed descriptions of experimental preparation and microelectrode mapping techniques can be found in previous publications (Kilgard and Merzenich, 1998a, 2002, 1998b; Kilgard et al., 2001a,b) and are described in brief below.

\subsection{Preparation}

Nine pentobarbital anesthetized Sprague-Dawley rats ( $\sim 300$ grams) were implanted with platinum bipolar stimulating electrodes (2.3 posterior, 3.3 lateral and 7 $\mathrm{mm}$ ventral to bregma) into the right nucleus basalis using sterile stereotaxic techniques approved by the UT-Dallas animal care committee. After two weeks of recovery, each animal received 300-400 daily pairings of either tones or modulated noise with NB stimulation for 20-25 days. Daily pairing sessions lasted 3-4 h. The interval between successive pairings was pseudorandom between 12 and $80 \mathrm{~s}$.

Four rats heard two different randomly interleaved tones $(2 \mathrm{kHz}$ at $65 \mathrm{~dB}$ SPL and $14 \mathrm{kHz}$ at $55 \mathrm{~dB}$ SPL; $250 \mathrm{~ms}$ duration with $5 \mathrm{~ms}$ on/off ramps) paired with nucleus basalis stimulation. The tone frequencies and intensities were selected to activate two non-overlapping neural populations at different times. Five additional rats heard pulsed white noise $(15 \mathrm{~Hz}$ trains of six 25 $\mathrm{ms}$ white noise bursts; $5 \mathrm{~ms}$ ramps; $55 \mathrm{~dB}$ SPL) paired with NB stimulation to provide an input pattern in which there was sequential repetitive input that simultaneously activated the same high and low frequencies sectors of the tonotopic map and to ensure the observed results depend upon tonal activation. To mimic earlier experiments using amplitude modulated sounds, animals exposed to pulsed noise were also exposed to a $25 \mathrm{~ms}$ long noise burst at the same intensity and regularity that was not paired with NB activation (Kilgard and Merzenich, 1998b). Electrical stimulation of nucleus basalis consisted of 20 charge-balanced biphasic pulses ( $0.1 \mathrm{~ms}$ pulse width, $100 \mathrm{~Hz}, 200 \mathrm{~ms}$ train duration) that terminated with the end of each paired sound. For the four rats that heard randomly interleaved tones, electrical stimulation began $50 \mathrm{~ms}$ after tone onset. When pulsed white noise was paired with nucleus basalis activity, stimulation occurred simultaneously with the onset of the fourth noise burst in the train. In the animals implanted with stimulating electrodes, the current level $(70-180 \mu \mathrm{A})$ was chosen to be the minimum necessary to desynchronize the EEG during slow-wave sleep for 
1-2 s. Auditory and electrical stimuli did not evoke any observable behavioral responses, i.e. do not cause rats to stop grooming, or awaken, if sleeping.

\subsection{Electrophysiology}

Twenty-four hours after the last pairing session, animals were pentobarbital anesthetized $(50 \mathrm{mg} / \mathrm{kg})$, the right auditory cortex was surgically exposed, and multiunit neuronal responses were recorded (Brainware Software, Tucker-Davis Technologies) with parylene-coated tungsten microelectrodes inserted to a depth of $\sim 550 \mu \mathrm{m}$ (layers IV/V). Similar recordings were also obtained from fifteen experimentally naïve rats. As in our earlier experiments, potentials above approximately $0.18 \mathrm{mV}$ were considered to be action potentials. Electrocardiography and pulse oximetry were used to monitor circulatory function and depth of anesthesia. In addition, body temperature was monitored with a rectal probe and maintained at $37^{\circ}$ with a heating pad (FHC). The average length of each acute mapping experiment was $23.4 \mathrm{~h}$. The durations for experimental and naive control animals were not significantly different. Microelectrode penetration sites were chosen to avoid damaging blood vessels while generating a detailed and evenly spaced cortical map. All auditory stimuli were presented free-field with
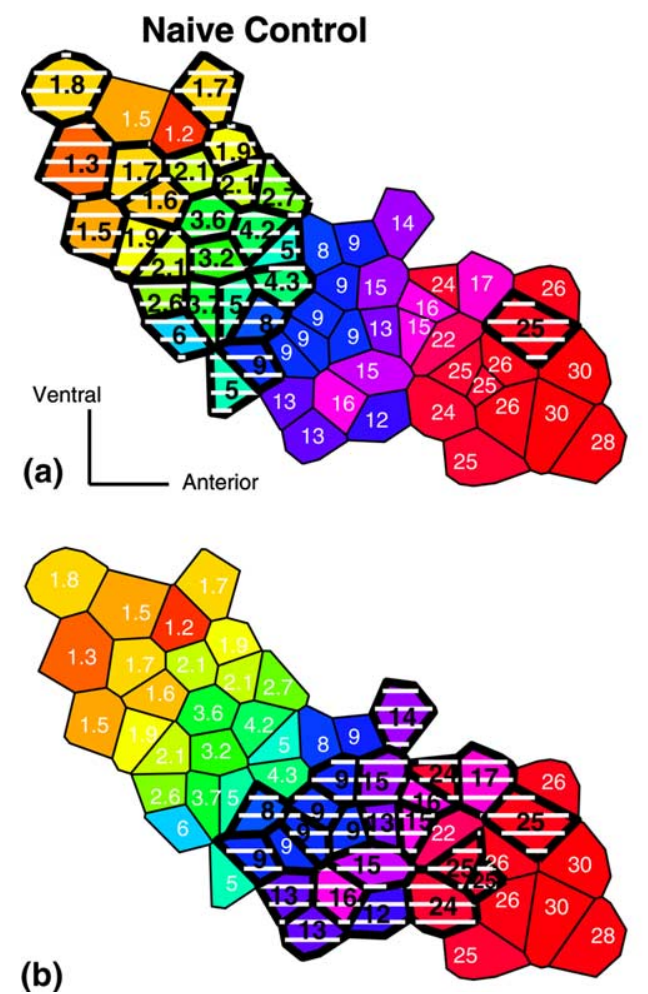

(d)

(c)

the same speaker used during the nucleus basalis-sound pairings. For each recording site, complete frequencyintensity response areas (receptive fields) were constructed from the responses to 1296 different tonal stimuli presented in a pseudorandom sequence over 16 intensities and 81 frequencies. All tonal stimuli used during the acute mapping phase of this study were $25 \mathrm{~ms}$ long.

In the animals exposed to noise bursts and in a subset of the naive controls, repetition rate transfer functions were also derived at each site by presenting a sequence of noise bursts. Noise burst trains contained six $75 \mathrm{~dB}$ noise bursts (each noise burst was $1.2-30 \mathrm{kHz}$ bandwidth and $25 \mathrm{~ms}$ in duration) presented at 14 repetition rates ranging from 3 to $19 \mathrm{~Hz}$.

\subsection{Data analysis}

Primary auditory cortex was defined on the basis of its short latency $(8-25 \mathrm{~ms})$ responses and its continuous tonotopy, and boundaries were determined using nonresponsive and non-primary sites (i.e., sites exhibiting long-latency responses, unusually high thresholds, or very broad tuning) (as in Kilgard and Merzenich, 1999). Characteristic frequency (CF), threshold, and latency were determined using a blind procedure. $\mathrm{CF}$ is the frequency that evokes a consistent neural response

\section{Experimental (2 \& $14 \mathrm{kHz}$ w/NB)}
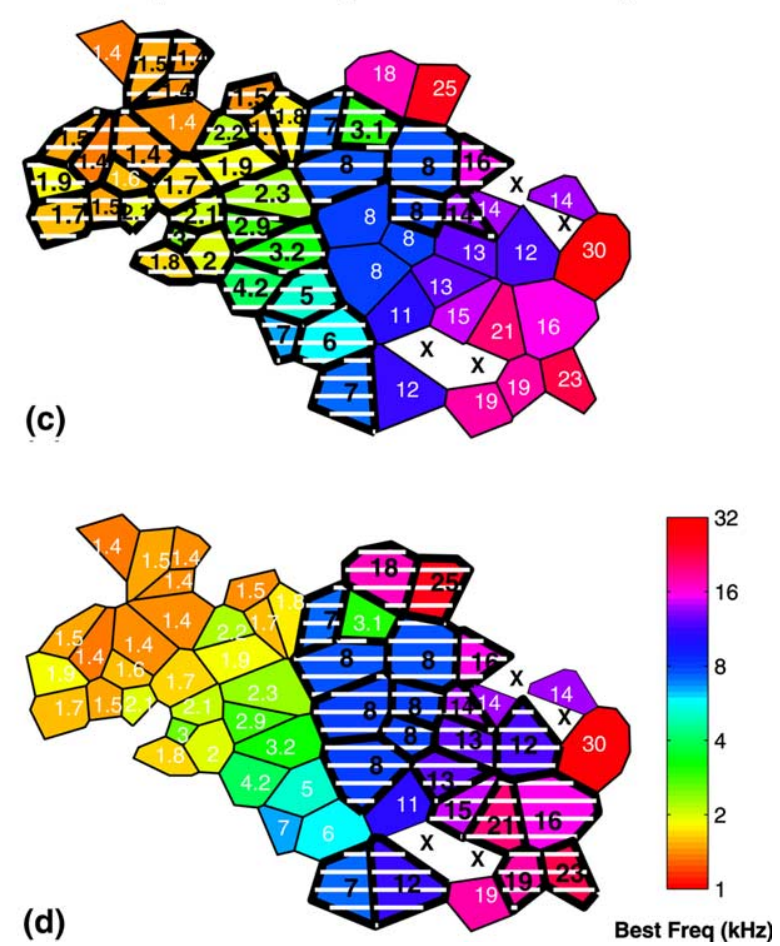

Fig. 1. Representative best frequency map of primary auditory cortex from a nä̈ve adult rat (a and b) and an animal conditioned with nucleus basalis activation separately paired with 2 and $14 \mathrm{kHz}$ tones $300-400$ times daily for one month (c and d). Each polygon represents one microelectrode penetration and color represents the best frequency. The objectively defined characteristic frequency of each location is also labeled within each polygon. White hatched regions with bolded black borders indicate regions activated by $2 \mathrm{kHz}$ at $65 \mathrm{~dB}(\mathrm{a}$ and c) and $14 \mathrm{kHz}$ at $55 \mathrm{~dB}(\mathrm{~b}$ and d) for the naive and experimental animal. Locations marked with $\mathrm{X}$ indicate sites where a reliable frequency could not be objectively defined. In (d), two $14 \mathrm{kHz}$ sites not activated had thresholds at 60 and $57 \mathrm{~dB}$. The line length on the direction marker in (a) shows the scale (0.25 mm). 
at the lowest stimulus intensity. The minimum latency was defined as the time from stimulus onset to the earliest consistent response for any of 15 intensities of the three frequencies that were nearest the $\mathrm{CF}$ (45 stimuli). Peak latency was quantitatively determined as the time to reach peak response in the PSTH while termination of response was defined as the time after stimulus onset at which activity returned to baseline levels. Response duration was defined as the time from the start of the response (i.e., minimum latency) to the end of the peak in the PSTH. Spontaneous activity is estimated as the number of spikes occurring in the first $5 \mathrm{~ms}$ after onset of the 1296 tones. The response strength of each site was estimated by determining the average number of spikes in response to tones of every frequency and intensity combination that fell within its receptive field.

Cortical response maps were reconstructed using the Voronoi tessellation procedure (Kilgard and Merzenich, 1999; Read et al., 2001) (see Fig. 1). These maps were used to quantify changes in the percent of cortex representing stimulus features. The tessellation procedure generates polygons from a set of non-uniformly spaced points so that every point in the polygon was nearer to the sampled point than to any other. This permits area information to be estimated from a number of discretely sampled recording sites by assigning each point on the cortical surface the qualities of the closest sampled point. The area measures generate reliable estimates of the percent of primary auditory cortex that is responsive to a given frequency-intensity combination. An advantage of this measure is that it allows higher sampling of cortical regions of interest without introducing bias into group data because densely sampled regions result in smaller polygons that contribute less to the overall percent. This technique is valuable because it can be used to estimate the magnitude of topographic reorganization (as in Kilgard and Merzenich, 1998a).

Due to the apparent regional specificity observed in reorganization of the tonotopic maps, response characteristics of cortical sites from all animals were subdivided into three 1.4 octave frequency ranges: low (sites with $\mathrm{CFs}$ between 1.2 and $3.3 \mathrm{kHz}$ ), medium (CFs between 3.3 and $8.6 \mathrm{kHz}$ ), and high (CFs between 8.6 and $22.8 \mathrm{kHz}$ ) and analyzed separately. The low and high frequency bands were centered on the frequencies ( 2 and $14 \mathrm{kHz}$ ) paired with nucleus basalis stimulation.

Best repetition rate was defined as the repetition rate that elicited the greatest number of evoked spikes. In this study, statistical significance was assessed using two tailed $t$ tests.

\section{Results}

The physiological data presented here were obtained from over 1100 recording sites in primary auditory cor- tex from 24 rats. Neural responses from four rats exposed to tones separated by 2.8 octaves and five rats exposed to noise bursts were compared to 15 experimentally naive controls. The pattern of neural activity generated by randomly interleaved high and low frequency tones led to: (1) modest reorganization of the frequency map; (2) regionally specific decreases in cortical excitability; (3) regionally specific increases in spontaneous firing rate activity; and (4) longer response latencies, when compared to naïve control animals.

\subsection{Frequency map plasticity}

Two and $14 \mathrm{kHz}$ tones activate distinct non-overlapping regions of the A1 frequency map. Fig. 1(a) and (b) illustrates the best frequency map of an experimentally naive animal. The highlighted recording sites in Panel $\mathrm{A}$ and $\mathrm{B}$ responded to $2 \mathrm{kHz}(65 \mathrm{~dB}$ SPL $)$ and $14 \mathrm{kHz}$ ( $55 \mathrm{~dB}$ SPL) tones. The percent of A1 surface area (see Section 2) that responded to the $2 \mathrm{kHz}$ tone and to the $14 \mathrm{kHz}$ tone was $44 \%$ and $34 \%$, respectively. After exposure to both tones independently paired with nucleus basalis activation, more A1 recording sites responded to each of these tones (Fig. 1(c) and (d)). In the representative example from an experimental animal, $54 \%$ of A1 surface area responded to the $2 \mathrm{kHz}$ tone and $45 \%$ responded to the $14 \mathrm{kHz}$ tone. These results indicate that NB stimulation increases the proportion of A1 neurons that include the paired tones in their receptive fields.

Average map reorganization for each group was quantified by estimating the percent of cortex responding to tones ranging from 1 to $32 \mathrm{kHz}$ and 10 to 75 dB SPL for naive control and experimental animals (Fig. 2(a) and (b)). For example, in controls, the average percent of cortex responding to $30 \mathrm{~dB} 2 \mathrm{kHz}, 60 \mathrm{~dB} 2$ $\mathrm{kHz}$, and $60 \mathrm{~dB} 8 \mathrm{kHz}$ tones were $15 \% \pm 2.0$, $44 \% \pm 2.3$, and $45 \% \pm 2.9$, respectively. After pairing high and low frequency tones with nucleus basalis activation, a greater proportion of neurons in primary auditory cortex responded to frequencies just above $14 \mathrm{kHz}$ and below $2 \mathrm{kHz}$ (Fig. 2(b)). The percent of A1 responding, for example, to a $60 \mathrm{~dB} 1.6 \mathrm{kHz}$ tone and a $50 \mathrm{~dB} 15$ $\mathrm{kHz}$ tone increased significantly $(p<0.05)$ from $42 \% \pm 2.4$ and $35 \% \pm 1.9$ to $52 \% \pm 9.5$ and $44 \% \pm 6.0$, respectively. These changes represent a $25 \%$ increase in the number of neurons that respond to these frequencies. The remodeling of the frequency maps after experience with tones that asynchronously activated the high and low frequency regions of A1 is best seen by calculating the difference in the percent of cortex responding to each tone from naive controls (Fig. 2(c)). The increased responses to tones near the paired frequencies indicate that competing, asynchronous tonal inputs distorted the normal frequency representation, apparently in a frequency specific manner. No significant changes in 

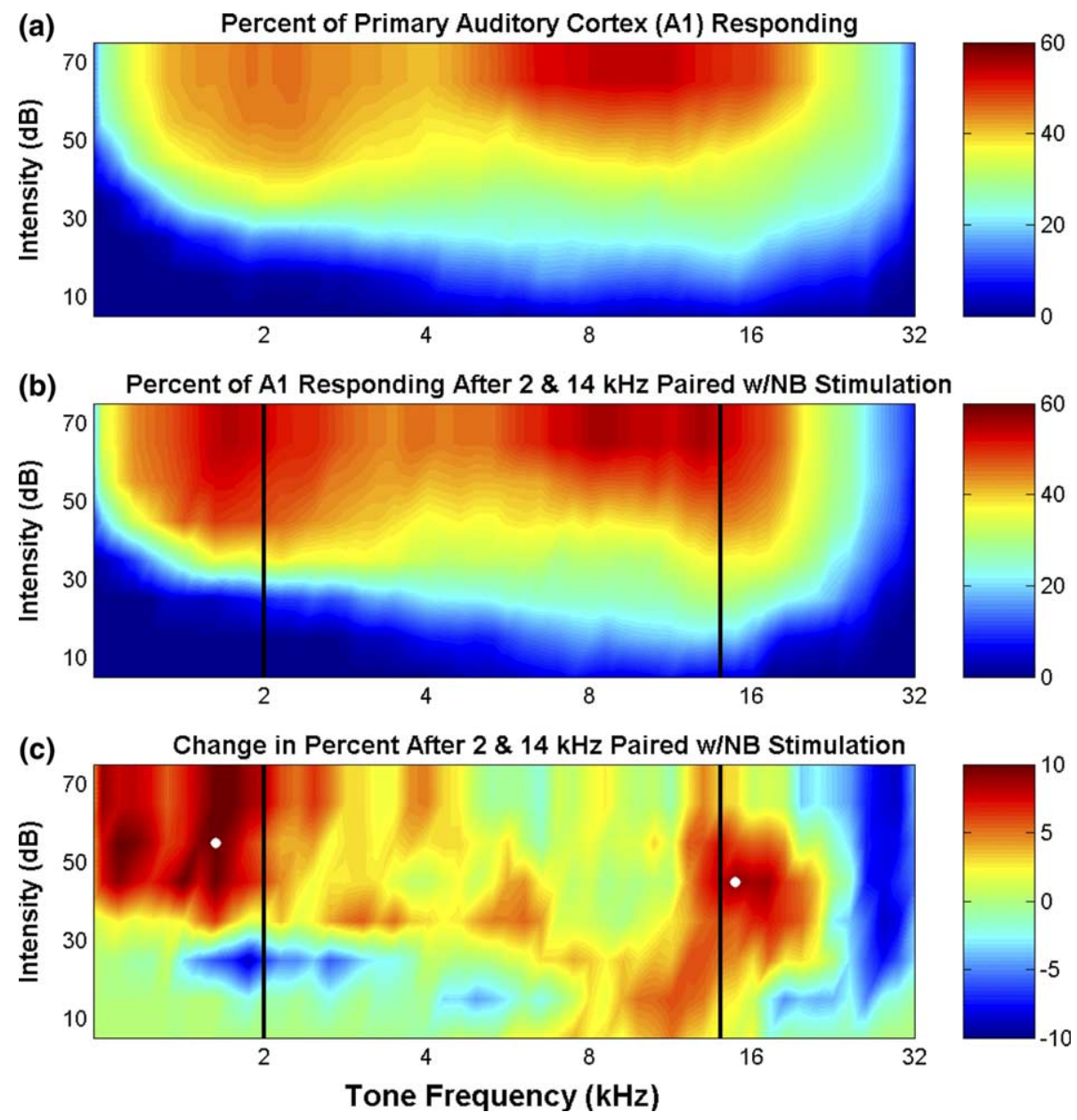

Fig. 2. Summary of frequency map plasticity. ( $a$ and $b$ ) The percent of the surface of primary auditory cortex (A1) that responds to each tone is represented as a color in experimentally näve rats $(\mathrm{a}, n=15)$ and those conditioned with 2 and $14 \mathrm{kHz}$ tones paired with nucleus basalis (NB) stimulation (b, $n=4)$. The percent of $\mathrm{A} 1$ responding was quantified as the sum of the areas of all the recording sites that responded to the tone frequency-intensity combination of interest divided by the total area of A1 (as in Kilgard and Merzenich, 1998a). (c) The difference in the percent cortical area of the naive group and the conditioned group illustrates the magnitude of the cortical map reorganization. Solid vertical lines (b and c) mark the tone frequencies paired with nucleus basalis activation for the group conditioned with asynchronous tonal inputs. The white dots in (c) indicate frequency-intensity combinations (described in the text) that were statistically different between the naive controls and experimental animals.

receptive field size were observed $(1.42 \pm 0.03$ octaves vs. $1.44 \pm 0.04$ octaves at $20 \mathrm{~dB}$ above threshold for naives and animals exposed to two tones, $p>0.5$ ).

\subsection{Effect of asynchronous inputs on response strength}

The finding of regional changes in frequency preference suggests that other response parameters may also have been altered in these regions. After exposure to 2 and $14 \mathrm{kHz}$ tones reinforced by nucleus basalis stimulation, both the high and low frequency bands showed significant changes in response strength (Fig. 3). The response of neurons in the low frequency band was decreased by $20 \%$ and the response of neurons in the high frequency band was decreased by $15 \%$. No difference was observed in the average response strength of experimental and control sites from the intermediate region (CFs $3.3-8.6 \mathrm{kHz}$ ).

\subsection{Effect of asynchronous inputs on neural threshold}

The regional decrease in response strength was accompanied by a regional decrease in intensity threshold. Fig. 4 shows the changes in neural sensitivity for each of the three frequency bands. The average threshold in the high and low frequency regions were increased by nearly $4 \mathrm{~dB}$, while no significant difference was observed in the intermediate region. The frequency-specific increase in threshold and decrease in response strength indicate that spectrally distinct input patterns can reduce cortical excitability in a topographically specific manner.

\subsection{Effect on spontaneous activity}

Spontaneous firing rate (see Section 2) was also altered in a regionally specific manner. While there were no changes in the high and low frequency regions, we 


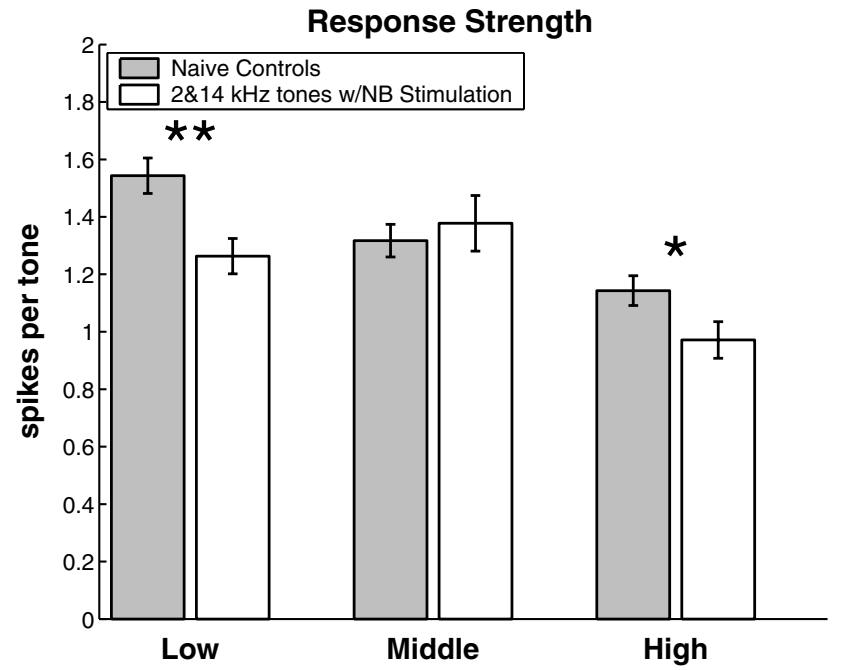

Fig. 3. Frequency specific decrease in response strength (mean \pm SEM) due to asynchronous activation of the high and low frequency regions of A1. Data are pooled for all recording locations in the low frequency band (CFs between 1.2 and $3.3 \mathrm{kHz} ; 161$ control sites and 93 experimental sites), the middle frequency band (CFs between 3.3 and $8.6 \mathrm{kHz} ; 158$ control sites and 75 experimental sites), and the high frequency band (CFs between 8.6 and $22.8 \mathrm{kHz}$; 193 control sites and 99 experimental sites). ${ }^{*}$ Statistical significance at $p<0.05 ; * *$ statistical significance at $p<0.005$.

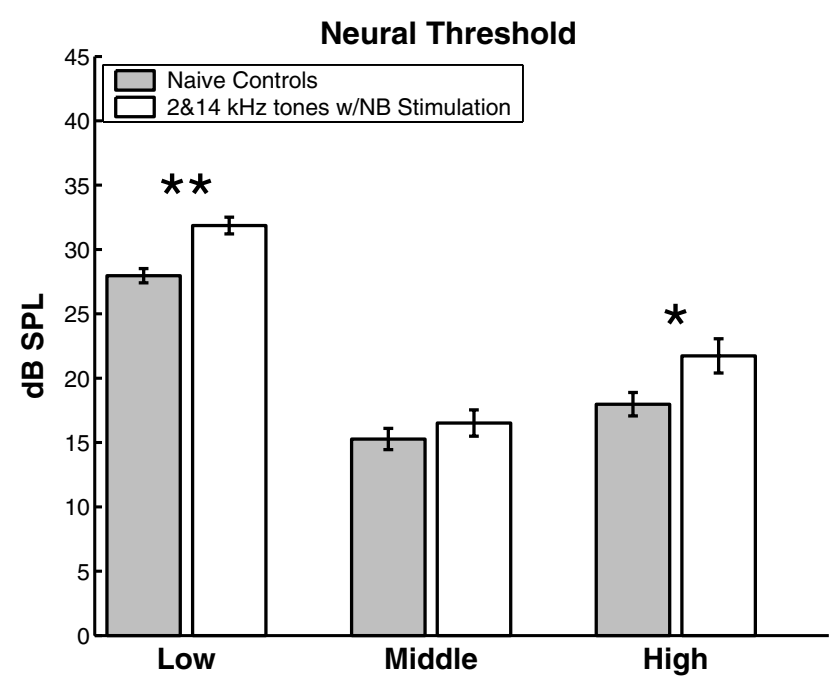

Fig. 4. Frequency specific increase in neural threshold due to asynchronous activation of the high and low frequency regions of A1. Data are pooled using the convention described in Fig. 3. *Statistical significance at $p<0.05 ; * *$ statistical significance at $p<0.00001$.

observed a $67 \%$ increase in the spontaneous firing rate in the middle frequency region (Fig. 5).

\subsection{Response latency}

Neural response latency includes delays due to conduction, synaptic transmission, and synaptic integra-

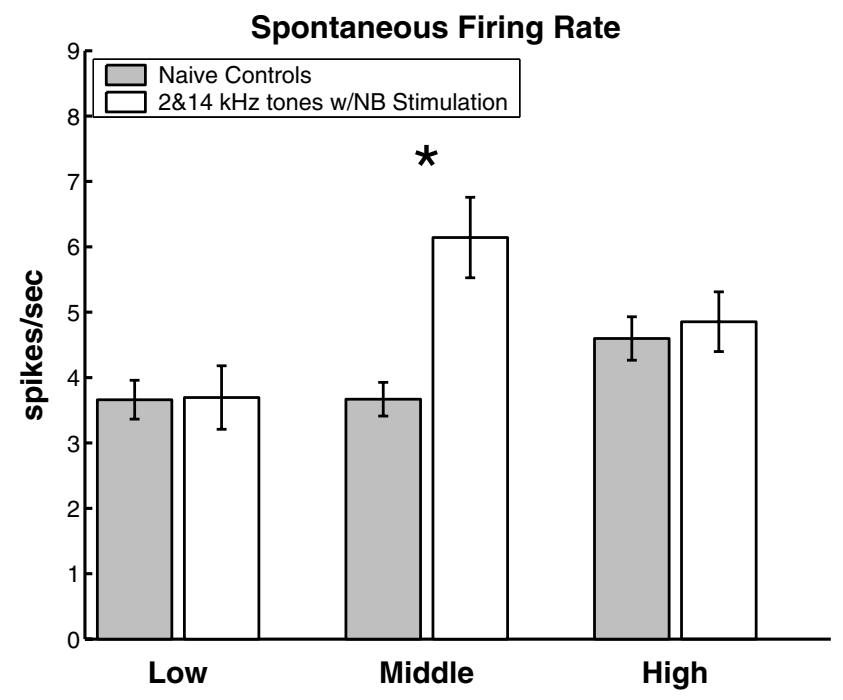

Fig. 5. Frequency specific increase in spontaneous firing rate activity due to asynchronous activation of the high and low frequency regions of A1. Data are pooled using the convention described in Fig. 3. *Statistical significance at $p<0.00005$.

tion. Although conduction velocity is unlikely to change significantly, previous studies have demonstrated that some aspects of cortical response latency are plastic. High and low frequency tones paired with nucleus basalis activation significantly increased the average time to first spike, peak response, and end of response all across the A1 frequency map, compared to naive controls (Fig. $6)$. The average minimum latency was lengthened from 13.8 to $15.2 \mathrm{~ms}(p<0.000001)$. The average time to peak for animals conditioned with these discrete inputs was nearly $4 \mathrm{~ms}$ longer than naive controls ( 22.6 vs. 18.8 $\mathrm{ms}, p<0.000005)$. The average response duration of

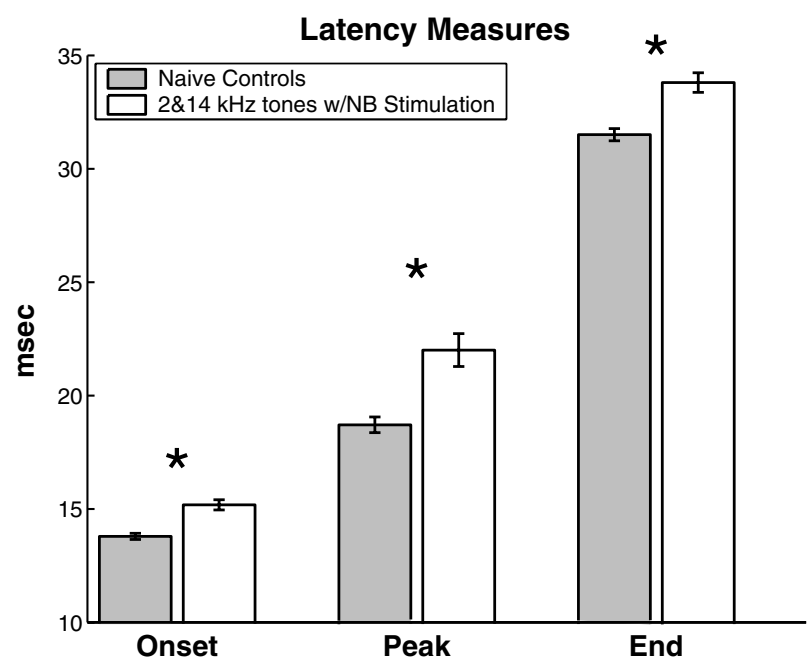

Fig. 6. Global increase in neural response latency due to asynchronous activation of the high and low frequency regions of A1. Data are pooled for all A1 recording locations (630 control sites and 318 experimental sites). ${ }^{*}$ Statistical significance at $p<0.00001$. 
the experimental animals was longer than naive controls (18.6 vs. $17.7 \mathrm{~ms}, p<0.05$ ).

\subsection{Input specificity}

To demonstrate that the observed plasticity does not occur after nucleus basalis stimulation is paired with any arbitrary sound containing high and low frequency energy, we paired white noise bursts with nucleus basalis stimulation in a different group of rats. Pairing NB stimulation with sounds that simultaneously activated neurons across A1 did not alter the frequency map. In contrast to the two tone group, there was no difference in the proportion of A1 neurons that responded to frequencies above $14 \mathrm{kHz}$ and below $2 \mathrm{kHz}$. The percent of $\mathrm{A} 1$ responding to a $60 \mathrm{~dB} 1.6 \mathrm{kHz}$ tone and a 50 dB $15 \mathrm{kHz}$ tone was indistinguishable between näve controls animals exposed to modulated noise $(42 \% \pm 2.4$ vs. $41 \% \pm 1.9$ and $35 \% \pm 1.9$ vs. $37 \% \pm 4.8$, $p>0.5)$.

Pairing noise bursts with NB stimulation did not significantly alter response strength or thresholds in any region of $\mathrm{A} 1(p>0.5)$. Noise pairing also did not alter any of the measures of response latency $(p>0.5)$. The ability to respond to successive inputs was unaffected (best repetition rate $=7.2 \pm 0.2$ and $7.4 \pm 0.2 \mathrm{~Hz}$ for controls and experimentals, respectively). Interestingly, spontaneous firing rate was altered in a regionally specific manner in this experimental group. In the middle frequency region spontaneous activity was increased by $48 \%$ ( $3.7 \pm 0.26$ vs. $5.4 \pm 0.25, p<0.005)$, while no increase was observed in the low and high frequency regions of A1 $(p>0.5)$. Pairing NB stimulation with noise bursts did not significantly alter the relationship between $\mathrm{CF}$ and anterior-posterior position (mean correlation coefficients $=0.923 \pm 0.016$ and $0.914 \pm 0.014$ for controls and experimentals, respectively).

\section{Discussion}

Numerous studies have demonstrated that altered sensory experience can reorganize cortical responses (Ahissar et al., 1992; Edeline, 1999; Engineer et al., 2004). Significant changes have been observed in receptive field size, cortical topography, response latency, spectrotemporal selectivity, response strength, and neuronal discharge coherence. Although it is clear that different behavioral paradigms generate different cortical changes, it has not been established what specific aspects of these experiences are responsible for the different forms of cortical plasticity. Studies of experiencedependent plasticity often differ in a number of parameters likely to be important for determining the form of plasticity: including input statistics, modality, behavioral response, task difficulty, task goal, motiva- tion, duration of training, background stimuli, and species. We have found that differential sensory experience associated with activation of nucleus basalis can generate systematic and enduring alterations in the spatial and temporal responses of primary auditory cortex neurons (Kilgard et al., 2001a). This preparation allows for direct comparison of many different forms of experience-dependent plasticity since all other experimental parameters can be held constant.

In the present study, we recorded responses of cortical neurons after several weeks of electrical activation of nucleus basalis paired with high and low frequency tones that asynchronously activated distinct regions of the tonotopic map. We report that this exposure leads to: (1) cortical map plasticity; (2) decreased cortical excitability; and (3) increased response latency. None of these changes have been reported to occur with nucleus basalis stimulation alone or tone exposure alone (Bao et al., 2003; Recanzone et al., 1993).

\subsection{Map and receptive field plasticity}

When a single unmodulated tone was repeatedly paired with activation of nucleus basalis the number of neurons representing the paired tone was increased by approximately 50\% (Kilgard and Merzenich, 1998a). When we paired two nearby tones (1.1 octave separation) the average map expansion was more modest $(\sim 25 \%)$. The decrease in the extent of cortical map expansion in a prior study when two nearby tones were associated with nucleus basalis stimulation may have resulted from competition for topographic sectors of the cortex between the two nearby inputs. In the current experiment the tones were separated by 2.8 octaves so that each tone activated distinct regions of the cortical map. It was theoretically possible that the additional separation would have made greater map expansion possible by reducing the competition for cortical space. The observed map reorganization was modest, as in our earlier two tone pairing study. This result suggests that map plasticity is reduced as the number of tones associated with NB stimulation increases. Consistent with this interpretation, no map reorganization was observed after seven different tones were independently paired with nucleus basalis stimulation (Kilgard et al., 2001a).

Average receptive field size can be either increased or decreased by operant training depending on task parameters (Recanzone et al., 1992a, 1993). Similar observations of differential plasticity have been made using NB stimulation paired with different sounds. Differences in the input statistics appear to be responsible for the direction and degree of receptive field plasticity. For example, frequency bandwidth (receptive field size) was significantly decreased when nearby tones (either 2 or 7) were associated with nucleus basalis (Kilgard et al., 2001a), and broadened when a single tone was 
repeatedly paired. In this study, we did not detect a significant change in receptive field size when two distant tones (2.8 octave separation) were associated with NB stimulation. This result suggests that receptive field narrowing develops when the cortical zones activated by each input overlap. This hypothesis is supported by our earlier observation that pairing seven different tones with NB stimulation generated receptive field narrowing in the absence of map plasticity.

\subsection{Changes in cortical excitability and latency}

Earlier studies showed that cortical excitability and latency could be increased or decreased depending on what sound was associated with NB stimulation. Pairing either an FM sweep or a train of simple tones increased response strength and decreased the mean onset latency (Kilgard et al., 2001a; Mercado et al., 2001; Moucha et al., in press). Pairing complex stimuli, such as a spectral ripple or a tone-tone-noise sequence, decreased latency without affecting response strength (Kilgard and Merzenich, 2002; Kilgard et al., 2001b). No changes in response strength or latency were noted after two nearby tones were paired with NB stimulation. Pairing seven different tones increased response latency (Kilgard et al., 2001a). These results suggested that cortical excitability and response latency are improved when sounds paired with NB activity activate a fixed cortical zone and are degraded when the sounds activate different zones at different times. After pairing NB stimulation with 2 and $14 \mathrm{kHz}$, most A1 neurons responded to one of two tones. It is not clear why the changes in response strength and threshold were restricted to low and high frequency regions of $\mathrm{A} 1$, while the changes in latency were not. It could be that response strength and threshold are altered only in neurons near the center of the region activated by the paired stimuli. Our observations of decreased excitability and increased latency when distant (but not nearby) tones were paired with NB supports our conclusion that asynchronous activation of non-overlapping cortical regions weakens cortical responses.

\subsection{Similarity with earlier plasticity studies}

Our observations that sensory input statistics determine the direction and extent of both cortical map plasticity and excitability are consistent with the conclusions of many earlier plasticity studies using very different techniques.

The topographic alterations observed above support the conclusions of several studies demonstrating that maps in primary sensory cortex tend to segregate inputs that are asynchronous and integrate correlated inputs (Clark et al., 1988; Hubel and Wiesel, 1965; Stryker and Strickland, 1984; Wang et al., 1995).
For example, fusion of two digits increases input correlation and leads to integration of inputs within the somatosensory cortical map (Allard et al., 1991; Clark et al., 1988). Surgical reversal restores natural input patterns and cortical map segregation. The normally distinct digit representations were also integrated together after extensive operant training with stimuli that simultaneously activate several fingers (Wang et al., 1995). In primary visual cortex, both strabismus and alternating electrical stimulation of the optic nerves disrupt the normally high correlation between the eyes and increase the segregation of inputs from each eye (Eschweiler and Rauschecker, 1993; Hubel and Wiesel, 1965; Roelfsema et al., 1994; Stryker and Strickland, 1984). These and other results suggest that simultaneous activation of neural networks leads to greater integration of sensory inputs, while asynchronous activation leads to segregation (Clark et al., 1988; Constantine-Paton and Law, 1978; Hubel and Wiesel, 1965; Stryker and Strickland, 1984; Wang et al., 1995).

Many studies have also shown that input correlations can shape cortical excitability, sensitivity, and latency. Increasing input correlation by strobe rearing, whisker pairing, or tactile discrimination training results in shorter latencies in visual and somatosensory cortices (Armstrong-James et al., 1994; Humphrey et al., 1998; Recanzone et al., 1992b). In contrast, decreasing input correlation via strabismus, monocular deprivation, or frequency discrimination training results in prolonged latencies in the visual and auditory cortices (Chino et al., 1988, 1983; Eschweiler and Rauschecker, 1993; Recanzone et al., 1993). Strabismus also decreases contrast sensitivity in visual cortex which may be analogous to the increased thresholds observed in the current study (Chino et al., 1988, 1983). Collectively, these observations are compatible with the hypothesis that synaptic correlation-based learning rules have a significant impact on the expression of network level plasticity (Buonomano and Merzenich, 1998).

\subsection{Potential mechanisms}

Changes in the relative balance of thalamocortical and corticocortical inputs could explain many of the changes observed in this and other studies. Recanzone and colleagues proposed that increased latency following extensive frequency discrimination training was due to a greater proportion of corticocortical connections, which have longer latencies than thalamocortical inputs. Recent findings in rat auditory cortex have shown that frequencies distant from the $\mathrm{CF}$ activate corticocortical inputs which generate subthreshold responses at long latencies (Kaur et al., 2004). A redistribution of synaptic inputs favoring corticocortical connections could explain the longer latencies and decreased response strengths ob- 
served in our study. The network consequences of experience-dependent plasticity likely depend on the local microarchitecture and cell type specific variation in synaptic plasticity rules, as well as the patterns of sensory stimulation and neuromodulator release.

Recent experiments demonstrating that input timing on the order of milliseconds can be the difference between synaptic strengthening and weakening provides a potential mechanism that would allow sensory statistics to precisely control the connectivity and temporal dynamics of large networks of neurons ( $\mathrm{Bi}$ and Poo, 1999; Feldman, 2000; Froemke and Dan, 2002; Schuett et al., 2001; Song and Abbott, 2001; Song et al., 2000; Yao and Dan, 2001; Zhang et al., 1998). Network models that incorporate this form of Hebbian learning, called spike-timing dependent synaptic plasticity (STDP), are highly sensitive to changes in input correlation (Song and Abbott, 2001). Tones generate the most spatially restricted auditory input and are distinct from most naturally occurring activation patterns. We propose that the focal activation pattern caused by tones coupled with the asynchronous activation of the high and low frequency regions of A1 resulted in a net decrease in the correlation across the frequency map compared to normal acoustic experience. This reduction in input correlation (unlike reductions in activity) may result in decreased synaptic strength (Song and Abbott, 2001) that could explain the longer latencies, higher thresholds, and lower response strengths that results from exposure to two distant tones.

\subsection{Experiential specificity}

To confirm that the effects reported above do not result from NB stimulation paired with any sound that activates both high and low frequency neurons, we also tested cortical responses from a group of rats that were exposed to noise bursts on an identical experimental schedule as the two tone group. This $15 \mathrm{~Hz}$ pulsed noise exposure had little effect on cortical responses compared to naive controls. Modulated broadband noises are quite common sounds in laboratory housing conditions and in natural settings. A possible reason that NB-noise pairing did not alter cortical topography, response strength, or latency is that this stimulus may not have differed sufficiently from naturally occurring input statistics.

The only response parameter that was significantly different from controls was the increased spontaneous activity of mid-frequency neurons. Since this effect was similar to the spontaneous changes observed after pairing two distant tones, the plasticity could simply be an effect of NB stimulation. However, changes in spontaneous activity are not always observed in animals exposed to nucleus basalis stimulation. Pairing a single tone did not significantly alter spontaneous activity in any region of A1 (Kilgard et al., 2001b). In contrast, spontaneous activity was reduced when seven different tones distributed across the rat frequency range were randomly interleaved and paired with nucleus basalis stimulation (Kilgard et al., 2001b). Collectively these nucleus basalis induced plasticity studies suggest that the pattern of acoustic experience may also be important in determining the level of spontaneous activity.

An earlier study reported that pairing NB stimulation with $10 \mathrm{~Hz}$ noise burst trains degraded topographic organization of primary auditory cortex (Bao et al., 2003). No such degradation was observed after NBnoise pairing in this study. It is reasonable to suspect that the different rates of modulation are responsible for the different results. While many cortical neurons can respond to every pulse of a $10 \mathrm{~Hz}$ train, very few can respond to every pulse of a $15 \mathrm{~Hz}$ train (Kilgard and Merzenich, 1999). Additional experiments will be needed to understand when NB-noise pairing will disorganize A1 topography.

\section{Conclusions}

Primary auditory cortex can be substantially reorganized in an experience-dependent manner. Results from a number of studies indicate that the spectral and temporal response properties of A1 neurons are shaped by the pattern of sensory inputs. The present study indicates that asynchronous high and low frequency tones paired with NB stimulation leads to cortical responses that are substantially slower, less sensitive, and less responsive. These inputs also induce modest reorganization of the frequency map that increases the segregation of high and low frequency responses. These results parallel observations in visual and somatosensory cortex suggesting that some aspects of network self-organization may be similar across modalities. The known physiological differences between the different primary sensory cortices may be influenced by differences in the input correlation structures of each modality. Since the complex relationship between sensory input patterns and cortical plasticity are still poorly understood, additional studies documenting how specific input patterns control cortical network organization and dynamics will be needed to clarify how neural plasticity mechanisms contribute to learning and memory in large populations of neurons.

\section{Acknowledgements}

This work was supported by NIH/NIDCD R03DC04534 (M.P.K.), an Individual NRSA Pre-Doctoral Award NIDCD F31-DC005285 (P.K.P.), and the American Academy of Audiology Student Investigator 
Award (P.K.P.). The authors thank Amber Cheney, Amanda Puckett, Wendy Dai, and Cherie Percaccio for assistance in animal training and colony management. We also acknowledge the constructive comments and suggestions of the reviewers.

\section{References}

Ahissar, E., Vaadia, E., Ahissar, M., Bergman, H., Arieli, A., Abeles, M., 1992. Dependence of cortical plasticity on correlated activity of single neurons and on behavioral context. Science 257, $1412-1415$.

Allard, T., Clark, S.A., Jenkins, W.M., Merzenich, M.M., 1991. Reorganization of somatosensory area $3 \mathrm{~b}$ representations in adult owl monkeys after digital syndactyly. J. Neurophysiol. 66, 10481058.

Armstrong-James, M., Diamond, M.E., Ebner, F.F., 1994. An innocuous bias in whisker use in adult rats modifies receptive fields of barrel cortex neurons. J. Neurosci. 14, 6978-6991.

Bakin, J.S., Weinberger, N.M., 1996. Induction of a physiological memory in the cerebral cortex by stimulation of the nucleus basalis. Proc. Natl. Acad. Sci. USA 93, 11219-11224.

Bao, S., Chang, E.F., Davis, J.D., Gobeske, K.T., Merzenich, M.M., 2003. Progressive degradation and subsequent refinement of acoustic representations in the adult auditory cortex. J. Neurosci. 23, 10765-10775.

Bi, G., Poo, M., 1999. Distributed synaptic modification in neural networks induced by patterned stimulation. Nature 401, 792796.

Bi, G., Poo, M., 2001. Synaptic modification by correlated activity: hebb's postulate revisited. Annu. Rev. Neurosci. 24, 139-166.

Buonomano, D.V., Merzenich, M.M., 1998. Cortical plasticity: from synapses to maps. Annu. Rev. Neurosci. 21, 149-186.

Chino, Y.M., Ridder 3rd, W.H., Czora, E.P., 1988. Effects of convergent strabismus on spatio-temporal response properties of neurons in cat area18. Exp. Brain Res. 72, 264-278.

Chino, Y.M., Shansky, M.S., Jankowski, W.L., Banser, F.A., 1983. Effects of rearing kittens with convergent strabismus on development of receptive-field properties in striate cortex neurons. J. Neurophysiol. 50, 265-286.

Clark, S.A., Allard, T., Jenkins, W.M., Merzenich, M.M., 1988. Receptive fields in the body-surface map in adult cortex defined by temporally correlated inputs. Nature $332,444-445$.

Conner, J.M., Culberson, A., Packowski, C., Chiba, A.A., Tuszynski, M.H., 2003. Lesions of the Basal forebrain cholinergic system impair task acquisition and abolish cortical plasticity associated with motor skill learning. Neuron 38, 819-829.

Constantine-Paton, M., Law, M.I., 1978. Eye-specific termination bands in tecta of three-eyed frogs. Science 202, 639-641.

Edeline, J.M., 1999. Learning-induced physiological plasticity in the thalamo-cortical sensory systems: a critical evaluation of receptive field plasticity, map changes and their potential mechanisms. Prog. Neurobiol. 57, 165-224.

Edeline, J.M., Hars, B., Maho, C., Hennevin, E., 1994. Transient and prolonged facilitation of tone-evoked responses induced by basal forebrain stimulations in the rat auditory cortex. Exp. Brain Res. 97, 373-386.

Engineer, N.D., Percaccio, C.R., Pandya, P.K., Moucha, R., Rathbun, D.L., Kilgard, M.P., 2004. Environmental enrichment improves response strength, threshold, selectivity, and latency of auditory cortex neurons. J. Neurophysiol. 92, 73-82.

Eschweiler, G.W., Rauschecker, J.P., 1993. Temporal integration in visual cortex of cats with surgically induced strabismus. Eur J. Neurosci. 5, 1501-1509.
Feldman, D.E., 2000. Timing-based LTP and LTD at vertical inputs to layer II/III pyramidal cells in rat barrel cortex. Neuron 27, 45-56.

Froemke, R.C., Dan, Y., 2002. Spike-timing-dependent synaptic modification induced by natural spike trains. Nature 416, 433438

Hebb, D.O., 1949. The Organization of Behavior. Wiley, New York.

Hubel, D.H., Wiesel, T.N., 1965. Binocular interaction in striate cortex of kittens reared with artificial squint. J. Neurophysiol. 28, 10411059.

Humphrey, A.L., Saul, A.B., Feidler, J.C., 1998. Strobe rearing prevents the convergence of inputs with different response timings onto area 17 simple cells. J. Neurophysiol. 80, 3005-3020.

Jenkins, W.M., Merzenich, M.M., Ochs, M.T., Allard, T., GuicRobles, E., 1990. Functional reorganization of primary somatosensory cortex in adult owl monkeys after behaviorally controlled tactile stimulation. J. Neurophysiol. 63, 82-104.

Kandel, E.R., 2001. The molecular biology of memory storage: a dialogue between genes and synapses. Science 294, 1030-1038.

Katz, L.C., Shatz, C.J., 1996. Synaptic activity and the construction of cortical circuits. Science 274, 1133-1138.

Kaur, S., Lazar, R., Metherate, R., 2004. Intracortical pathways determine breadth of subthreshold frequency receptive fields in primary auditory cortex. J. Neurophysiol..

Kilgard, M.P., Merzenich, M.M., 1998a. Cortical map reorganization enabled by nucleus basalis activity. Science 279, 1714-1718.

Kilgard, M.P., Merzenich, M.M., 1999. Distributed representation of spectral and temporal information in rat primary auditory cortex. Hear. Res. 134, 16-28.

Kilgard, M.P., Merzenich, M.M., 2002. Order-sensitive plasticity in adult primary auditory cortex. Proc. Natl. Acad. Sci. USA 99, 3205-3209.

Kilgard, M.P., Merzenich, M.M., 1998b. Plasticity of temporal information processing in the primary auditory cortex. Nature Neuroscience 1, 727-731.

Kilgard, M.P., Pandya, P.K., Engineer, N.D., Moucha, R., 2002. Cortical network reorganization guided by sensory input features. Biol. Cybern. 87, 333-343.

Kilgard, M.P., Pandya, P.K., Vazquez, J., Gehi, A., Schreiner, C.E., Merzenich, M.M., 2001a. Sensory input directs spatial and temporal plasticity in primary auditory cortex. J. Neurophysiol. 86, 326-338.

Kilgard, M.P., Pandya, P.K., Vazquez, J.L., Rathbun, D.L., Engineer, N.D., Moucha, R., 2001b. Spectral features control temporal plasticity in auditory cortex. Audiol. Neurootol. 6, 196-202.

Mercado, E., Bao, S., Orduna, I., Gluck, M.A., Merzenich, M.M., 2001. Basal forebrain stimulation changes cortical sensitivities to complex sound. Neuroreport 12, 2283-2287.

Metherate, R., Ashe, J.H., 1993. Nucleus basalis stimulation facilitates thalamocortical synaptic transmission in the rat auditory cortex. Synapse 14, 132-143.

Moucha, R., Pandya, P.K., Engineer, N.D., Rathbun, D.L., Kilgard, M.P., in press. Background sounds contribute to spectrotemporal plasticity in primary auditory cortex. Exp. Brain Res.

Rasmusson, D.D., Dykes, R.W., 1988. Long-term enhancement of evoked potentials in cat somatosensory cortex produced by coactivation of the basal forebrain and cutaneous receptors. Exp. Brain Res. 70, 276-286.

Read, H.L., Winer, J.A., Schreiner, C.E., 2001. Modular organization of intrinsic connections associated with spectral tuning in cat auditory cortex. Proc. Natl. Acad. Sci. USA 98, 8042-8047.

Recanzone, G.H., Merzenich, M.M., Jenkins, W.M., Grajski, K.A., Dinse, H.R., 1992a. Topographic reorganization of the hand representation in cortical area $3 \mathrm{~b}$ owl monkeys trained in a frequency-discrimination task. J. Neurophysiol. 67, 1031-1056.

Recanzone, G.H., Merzenich, M.M., Schreiner, C.E., 1992b. Changes in the distributed temporal response properties of SI cortical neurons reflect improvements in performance on a 
temporally based tactile discrimination task. J. Neurophysiol. 67, 1071-1091.

Recanzone, G.H., Schreiner, C.E., Merzenich, M.M., 1993. Plasticity in the frequency representation of primary auditory cortex following discrimination training in adult owl monkeys. J. Neurosci. 13, 87-103.

Roelfsema, P.R., Konig, P., Engel, A.K., Sireteanu, R., Singer, W., 1994. Reduced synchronization in the visual cortex of cats with strabismic amblyopia. Eur. J. Neurosci. 6, 1645-1655.

Schuett, S., Bonhoeffer, T., Hubener, M., 2001. Pairing-induced changes of orientation maps in cat visual cortex. Neuron 32, 325-337.

Song, S., Abbott, L.F., 2001. Cortical development and remapping through spike timing-dependent plasticity. Neuron 32, 339-350.

Song, S., Miller, K.D., Abbott, L.F., 2000. Competitive Hebbian learning through spike-timing-dependent synaptic plasticity. Nat. Neurosci. 3, 919-926.
Stent, G.S., 1973. A physiological mechanism for Hebb's postulate of learning. Proc. Natl. Acad. Sci. USA 70, 997-1001.

Stryker, M.P., Strickland, S.L., 1984. Physiological segregation of ocular dominance columns depends on the pattern of afferent electrical activity. Invest. Opthamol. Vis. Sci. Suppl. 25, 278.

Wang, X., Merzenich, M.M., Sameshima, K., Jenkins, W.M., 1995. Remodelling of hand representation in adult cortex determined by timing of tactile stimulation. Nature 378, 71-75.

Weinberger, N.M., 1998. Physiological memory in primary auditory cortex: characteristics and mechanisms. Neurobiol. Learn Mem. 70, 226-251.

Yao, H., Dan, Y., 2001. Stimulus timing-dependent plasticity in cortical processing of orientation. Neuron 32, 315-323.

Zhang, L.I., Tao, H.W., Holt, C.E., Harris, W.A., Poo, M., 1998. A critical window for cooperation and competition among developing retinotectal synapses. Nature 395, 37-44. 